\title{
Study on Merging and Optimization Method of Support Equipment Range in the Development Phase
}

\author{
Xiaoming Kang, Shihong Xiang \\ School of Automation Science and Electrical Engineering Beihang University, Beijing, China \\ Nanjing Glaway Software Co., LTD, China \\ x_kang@sohu.com, xiangshihong@glaway.com
}

\begin{abstract}
Keywords: Support Equipment range; Dynamic Programming; Optimization
Abstract. Two support equipment range merging optimization methods, i.e. direct optimization method and dynamic programming optimization method, were proposed in this paper. Direct optimization method is more suitable for simple systems with only limited types of support equipment, while dynamic programming optimization method works better for complex systems with a large number of types of support equipment. We gave out a case study to validate the dynamic programming optimization method. This paper provides theoretical support for resolving problem of optimizing support equipment range in the system development phase.
\end{abstract}

\section{Introduction}

Support equipment is one of the most important factors that affects system support effectiveness and support footprint. During system development phase, reasonable determination of support equipment range and optimizing support equipment quantity are important to reduce support footprint and support cost.

There have been several methods, for calculating support equipment demands during system development phase, such as Chen Jun and Chen Yonglong [1-2] provide a method of calculating approximate support equipment demands based on similar system theory or neural network model; Reference [3-8] provide the calculation model based on support activity processes modeling or queuing theory; While there are few literatures focusing on support equipment types merging method. Also Reference [9] just gives the process for determining support equipment types through supportability analysis, without support equipment types merging method, which is premise and basis for support equipment quantity determination. This paper proposes support equipment range merging criterion based on support function equivalence relation. Furthermore, this paper gives a basic merging optimization method and a multi-step merging optimization method based on dynamic programming theory. The multi-step merging optimization method provides theoretical support for optimizing support equipment types of some complex systems.

\section{Direct Optimization Method}

If the system complexity is not high and the types of support equipment are few, direct optimization method could be used to merge the support equipment types. Firstly, the optimization goal and objective function should be defined, then judging whether the support equipment types can be merged through support functional equivalence relationship of the support equipments. Support functional equivalence relationship is defined as a case, in which, at given condition, different support equipments' functions can meet a given supporting task requirement.

The set named $\mathrm{M}$ need be established to represent support task requirements, the set named $\mathrm{T}$ need to be established to represent support equipment types. The mapping between $\mathrm{R}$ and $\mathrm{P}$ by support function equivalence relation should be established finally, see Fig.1. 


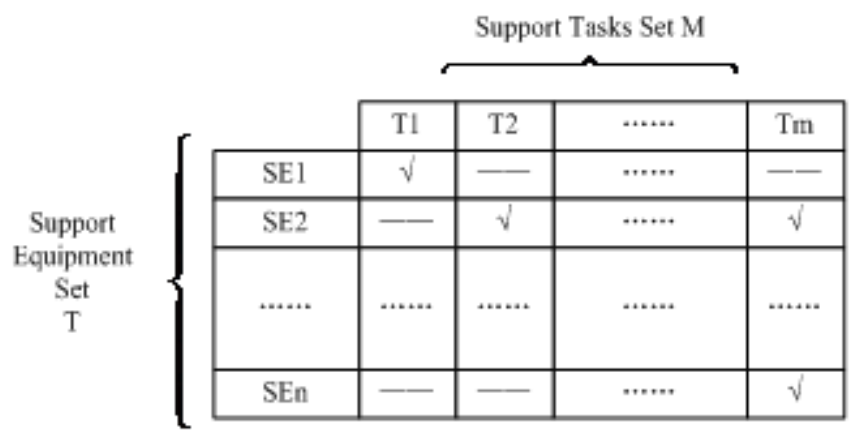

Fig.1 Mapping Relationships Between Set T and M

According to the mapping relationship, matrix $\mathrm{R}$ is generated, with $\mathrm{n}$ rows (the number of support equipment types) and m columns (the number of support tasks) consistent of elements 0 and 1.

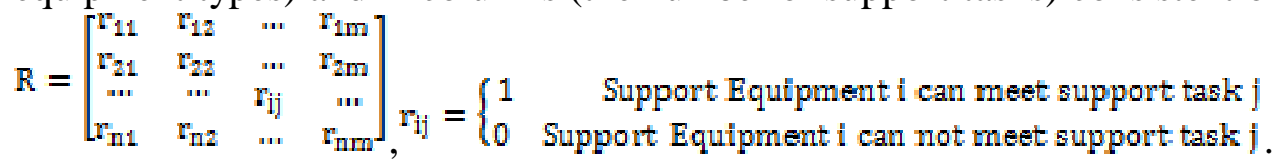

Given: Judgment matrix of merging is $\mathrm{D}$, it is also a $\mathrm{n}$ rows $\mathrm{m}$ columns matrix consistent of elements 0 and 1.

$$
\begin{aligned}
& D=\left[\begin{array}{cccc}
d_{11} & d_{12} & \ldots & d_{1 m} \\
d_{11} & d_{22} & \ldots & d_{2 m} \\
\ldots & \ldots & d_{i j} & \ldots \\
d_{n 1} & d_{n 2} & \ldots & d_{n m}
\end{array}\right], d_{i j} \text { in matrix D can be calculated through Equation 1: } \\
& d_{i j}=\operatorname{MAX}\left(2 * r_{i j}-\sum_{k=1}^{m} n_{i k}, 0\right)
\end{aligned}
$$

If there is any row full of value 0 , the support equipment type represented by this row can be merged. After set optimization goal and objective function, the optimal support equipment range can be calculated through Equation 2.

The objective function is defined as:

$\int \mathrm{C}_{\mathrm{s}}=\mathrm{MIN}(\mathrm{f}(\mathrm{L}) \mid \mathrm{L} \in \mathrm{S})$

$\left\{\mathrm{f}(\mathrm{L})=\Sigma \mathrm{C}_{\mathrm{pm}+\mathrm{i}} \mid \mathrm{t} \in \mathrm{L}\right.$

Where, $\mathrm{C}_{\mathrm{s}}$ is the optimization goal(such as the lowest usage cost); $\mathrm{L}$ is the subset of $\mathrm{T}$ that meets support tasks requirements; $S$ is the collection of all $L ; f(L)$ is the function for calculating the accumulated goal(such as usage cost) of all support equipments in $\mathrm{L} ; \mathrm{C}_{\mathrm{pm}}$ is the objective value of support equipment t.

\section{Dynamic Programming Optimization Method}

Support tasks and support equipment types of complex system are various, so that it is very complicated to establish the support equipment equivalence relationship. The calculation amount of merging optimization through direct optimization method will be too huge. In this case, dynamic programming optimization method, which decomposes the optimization process into multiple steps, is more practicable.

The non-optimal support equipment combinations should be excluded before each step of optimization, then the remaining combinations can be directly optimized. The optimal construction can be achieved repeating the process. See Fig.2.

As Fig. 2 shown, all support equipment types were divided into three groups:SE1, SE2 and SE3, then decision principles through multi-step merging optimization process should be decided. At each step, support equipment types are combined as a state of this step. The set of all state is called state set, named S. The state set of step 1 is named S1, which contains unique element -- the first group of support equipment types. The state set of step 2, named S2, is get by excluding the non-optimal combinations of support equipment types in step 2 based on S1 and a certain strategy. The state set of step 3, named S3, is get by excluding the non-optimal combinations of support equipment types at step 3 based on S2 and a certain strategy. Since the third step is the last step, each element in S3 represents 
the possibly optimal combination of support equipment types. If the objective function is set to $\mathrm{V}$, the optimal support equipment catalogue can be determined based on S3 and V.

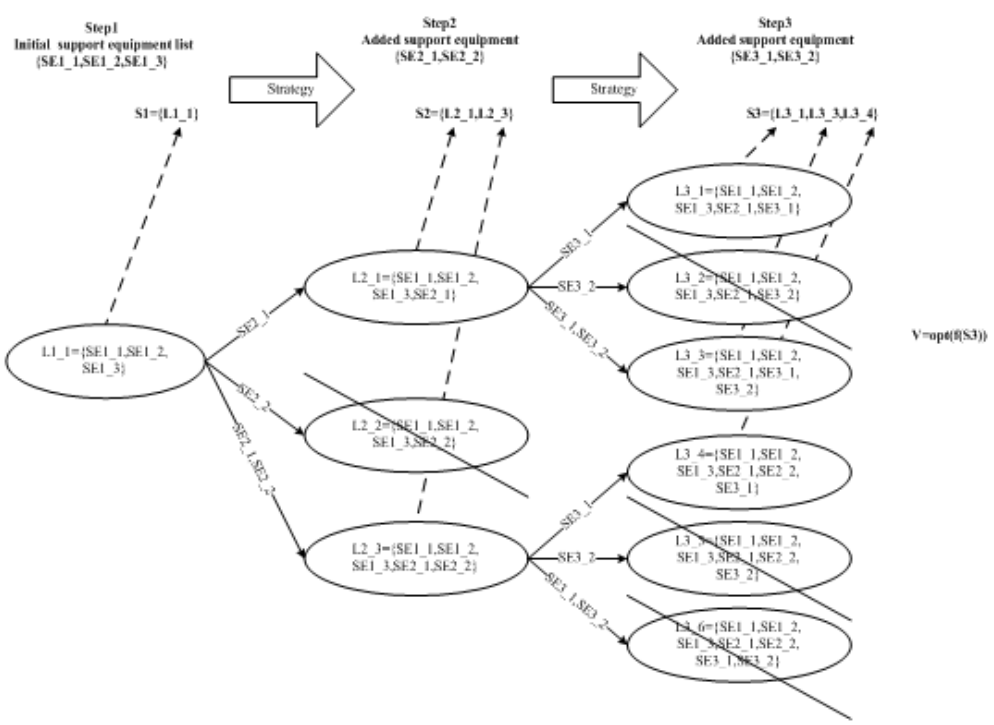

Fig.2 Multi-step merging optimization process

\section{Dynamic Programming Optimization Process}

The dynamic programming optimization process of support equipment catalogue contains 4 steps:

1.Determining the goal of support equipment range optimization;

2.Determining how many steps that the strategy of support equipment range optimization are devided into;

3.Establishing the strategy and determine the set of all possibly optimal combinations( state set) of support equipment types of each step ;

4.Determining the optimal combination of support equipment types in the state set of last step through the optimal objective function.

The optimization process is shown in Fig.3.

Before using dynamic programming optimization method, the goal of optimizing support equipment range must have been determined. This paper chooses support equipment usage cost(SEUC) as the optimization goal. The accumulated usage cost of each combination of support equipment types should be calculated to drive the next step.

The amount of steps after determining the optimization goal should be determined, then the functions and the tasks that all support equipments can be completed in each support equipment group should be determined. Finally, the relationship between the tasks and function requirements should be mapping. See Fig.4.

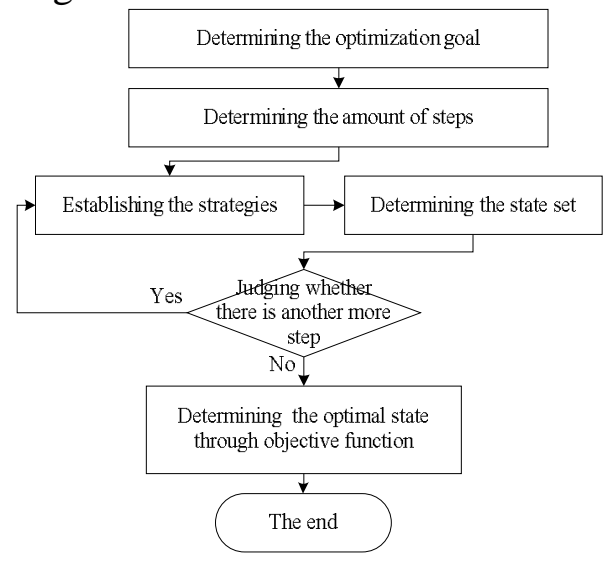

Fig3. Dynamic programming optimization process

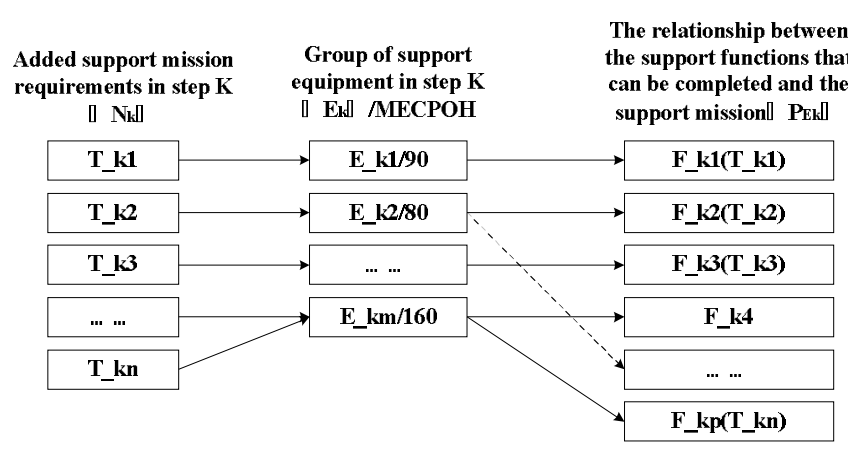

Fig.4 Mapping the relationship of support tasks, equipments and function requirements 
At each step, the combinations of support equipment types (states) that impossibly be the optimal combinations should be excluded according to some strategy. The strategy of each step consists two constraint conditions: 1)All combinations of support equipment types must satisfy the support function requirements; 2)At this step, there is a combination of support equipment types that can complete more function and has lower accumulated usage cost than any other combinations. The determination of equivalent relation is the basis of comparing which combination can complete the most functions. The strategy of step $\mathrm{k}$ is named $u_{\mathrm{k}}$, which is defined as:

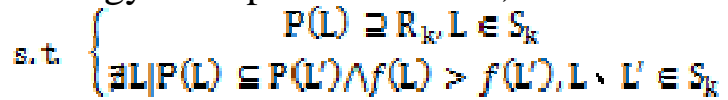

Where, $\mathrm{L}$ is the combination of support equipment types, $\mathrm{P}(\mathrm{L})$ is the support function that $\mathrm{L}$ can complete; $\mathbb{R}_{\mathrm{k}}$ is the support function requirements of the step $\mathrm{k}$; $\mathrm{S}_{\mathrm{kn}}$ is the state set of the step $\mathrm{k} ; f(\mathrm{~L})$ is the accumulated usage cost of $\mathrm{L}$.

$\mathbb{R}_{\mathrm{k}}$ is determined by:

$\left\{\begin{array}{cc}\mathbb{R}_{\mathrm{kg}}=\mathrm{R}_{\mathrm{k}-1} \cup \mathrm{N}_{\mathrm{kg}} & 1<k<n \\ \mathbb{R}_{1}=\mathrm{N}_{1} & \mathrm{k}=1\end{array}\right.$

where, $\mathrm{n}$ is the amount of steps; $\mathbb{N}_{\mathrm{k}}$ is the set of added support tasks.

The state is a combination of some support equipment types which satisfy the support mission requirements at current step. The state set is the set of all combinations and is defined as:

$\left\{\begin{array}{cc}S_{k}=T\left(S_{k-1}, u_{k}\right) & 1<k<n \\ S_{1}=\left[I_{1}\right] & k=1\end{array}\right.$

where, $\mathrm{n}$ is the amount of steps; $\mathbb{S}_{\mathrm{k}}$ is the state set after finishing the step $\mathrm{k}$, and each element of $\boldsymbol{S}_{\mathrm{k}}$ means a combination that satisfies the support task requirements of current step; $\mathbf{S}_{1}$ is the combination of support equipment types at the first step and has only one element $L_{1}$; $u_{k}$ is the strategy of step k.

The process of determining $S_{\mathrm{k}}$ is below:

Given $S_{k-1}=\left[L_{k-1_{1}}, L_{k-1_{2}}, \ldots, \mathbf{L}_{k-1_{m}}\right]$, and the grouping of support equipment types at step $\mathrm{k}$ is $\mathrm{E}_{\mathrm{k}}$. The union of all elements of $S_{\mathrm{k}-1}$ and all subsets of $\mathbf{E}_{\mathrm{k}}\left(\right.$ with $\mathbf{E}_{\mathrm{k}}$ itself $)$ is the temporary state set of step $\mathrm{k}$, named $S_{\mathrm{k} o}^{r}$ Using $\mathrm{u}_{\mathrm{k}}$ to make the decision of $S_{\mathrm{k}}^{r}$, then get the state set of step $\mathrm{k}$, named $S_{\mathrm{k}}$.

The last step(step $n$ ) is to solve the optimal objective function and acquire the optimal support equipment catalogue. The objective function is defined as:

$N_{s}=\operatorname{MiN}(f(\mathrm{~L}) \mid \mathrm{L} \in \mathrm{S})$

$f(\mathrm{~L})=\sum c_{p m r} \mid t \in \mathrm{L}$

Where, $V_{3}$ is the lowest accumulated SEUC; $S$ is the state set of the last step; $L$ is an any element of $S$; $\mathrm{f}(\mathrm{L})$ is the function by which to calculate the SEUC of all support equipments; $\mathrm{C}_{\mathrm{pm}}$ is the SEUC of support equipment $\mathrm{t}$.

The state L, which satisfies the optimal objective function and has the lowest accumulated SEUC, is the final result of support equipment types optimization.

\section{Case Study}

Given: A system is composed of 3 functional systems. Fig 5 7 show the initial support equipment range, the list of support tasks, the list of support functions that the initial support equipments can be completed, and the relationship of these three aspects.

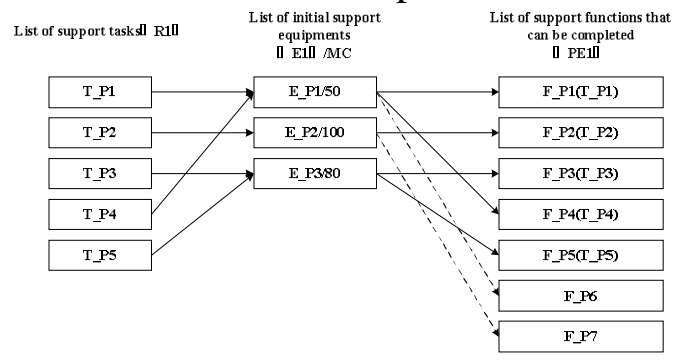

Fig. 5 Support relationship of System 1

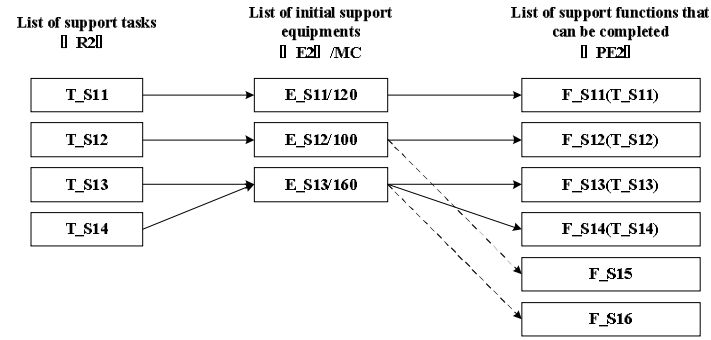

Fig.6 Support relationship of System 2 


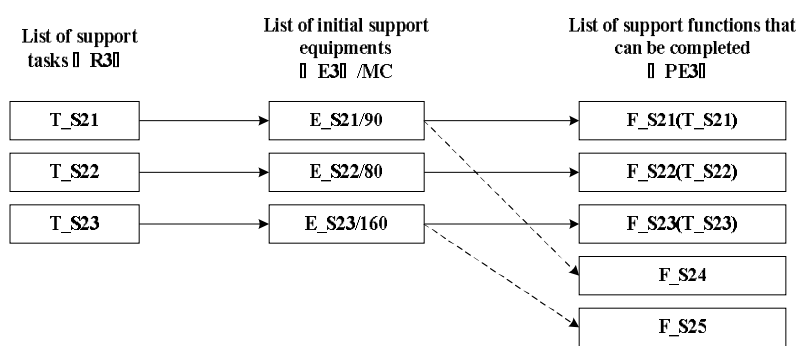

Fig.7 Support relationship of System 3

The list of equivalent relations is shown in Table 1.

Table. 1 The List of The Equivalent Relation

\begin{tabular}{|c|c|}
\hline Support Function & Equivalent Support Function \\
\hline F_P1 & F_S11 \\
\hline F_P6 & F_S12 \\
\hline F_P7 & F_S22 \\
\hline F_S15 & F_S23 \\
\hline
\end{tabular}

The optimization process should be divided into three steps to make decision, and each step decides the support equipments of one system.

The first step of making decision is using basic information about System 1 to determine state $\Sigma_{1}$ 、 $P$ 、 $\mathbb{R}_{1}$, there is:

$S_{1}=\left\{L_{1}\right\}=\left\{\left\{E_{-} P 1, E_{-} P 2, E_{-} P 3\right\}\right]$

$\mathrm{P}_{\mathrm{L} 1}=\left[\mathrm{F}_{-} \mathrm{P} 1, \mathrm{~F}_{-} \mathrm{P} 2, \mathrm{~F}_{-} \mathrm{P} 3_{2} \mathrm{~F}_{-} \mathrm{P} 4, \mathrm{~F}_{-} \mathrm{P} 5, \mathrm{~F}_{-} \mathrm{P} 6, \mathrm{~F}_{-} \mathrm{P7}\right]$

$\mathbb{R}_{1}=\left\{T_{-} P 1, T_{-} P 2, T_{-} P 3, T_{-} P 4, T_{-} P 5\right\}$

$=\left\{F_{-}\right.$P1, F $P 2, F_{-} P 3, F_{-}$P4,$F_{-}$P5 $\}$

The second step of making decision is using result of the first step and the basic information about System 2 to determine $\mathbb{R}_{2} 、 S_{2}$ 。

Firstly, calculate $\mathbb{R}_{2}$ :

$\mathbb{R}_{2}=\mathbb{R}_{2} \cup \mathbb{N}_{2}$

$=\left\{F_{-} P 1, F_{-} P 2, F_{-} P 3, F_{-} P 4, F_{-} P 5\right\}$

$U\left\{F_{-} S 11, F_{-} S 12, F_{-} S 13, F_{-} S 14\right\}$

$=\left\{F_{-}\right.$P1, F_P2, F_P3, F_P4, F_P5, F_S12, F_S13, F_S14\}

The subsets of $\mathbb{E}_{2}$ are $\left[\mathbb{E}_{-} S 11\right],\left\{\mathbb{E}_{-} \$ 12\right],\left[\mathbb{E}_{-} S 13\right],\left\{\mathbb{E}_{-} \$ 11, \mathbb{E}_{-} S 12\right\},\left[\mathbb{E}_{-} S 11, \mathbb{E}_{-} S 13\right],\left\{\mathbb{E}_{-} S 12, \mathbb{E}_{-} S 13\right]$ and $\left\{E_{-} S_{11}, E_{-} S_{12}, E_{-} S 13\right\}$. The union of these subsets and $\mathrm{L}_{1}$ is a new set. Judge the support functions $(\mathrm{P})$ that the new set can be completed whether satisfy the requirements of $\mathbb{R}_{\mathbf{2}}$. There are four sets that satisfy $\mathbb{R}_{2}: \quad\left\{E_{-} P 1, E_{-} P 2, E_{-} P 3, E_{-} S 13\right\}$ [E_P1, E_P2, E_P3, E_S11, E_S13]

$\left\{E_{-} P 1, E_{-} P 2, E_{-} P 3, E_{-}\right.$S12, E_S13\}, \{E_P1, E_P2, E_P3,E_S11, E_S12, E_S13\}. Made decision using the strategy $u$, then we can exclude $\left\{E_{-} P 1, E_{-}\right.$P2,E_P3,E_S11,E_S13] and [E_P1,E_P2,E_P3,E_S11,E_S12,E_S13] because they are impossible to be the optimal combinations. So $S_{2}$ contains two elements :

$S_{2}=\left\{I_{2_{1}}, I_{2_{2}}\right\}$

$\mathrm{L}_{2_{1}}=\left\{E_{-} P 1, E_{-} P 2, E_{-} P 3, E_{-} S 13\right\}$

$\mathrm{L}_{2_{2}}=\left\{E_{-}\right.$P1, E_P2, E_P3, E_S12, E_S13]

The third step of making decision is using result of the second step and the basic information about System 3 to determine $R_{2}, S_{z}$. Then calculate $V_{S a}$ and determine the optimal support equipment catalogue.

Firstly, calculate $\mathbb{R}_{z}$ :

$\mathbb{R}_{\mathrm{g}}=\mathbb{R}_{\mathrm{g}} \cup \mathrm{N}_{\mathrm{g}}$

$=\left\{F_{-} P 1, F_{-} P 2, F_{-} P 3, F_{-} P 4, F_{-} P 5, F_{-} S 12, F_{-} S 13, F_{-} S 14\right\}$

$U\left[F_{-} 521, F_{-} S 22, F_{-} S 23\right\}=\left\{F_{-} P 1, F_{-} P 2, F_{-} P 3, F_{-} P 4, F_{-} P 5\right.$,

$\left.F_{-} S 12, F_{-} S 13, F_{-} S 14, F_{-} S 21, F_{-} \$ 22, F_{-} \$ 23\right]$ 
The subsets of $E_{2}$ are $\left\{E_{-} S 21\right\},\left\{E_{-} S 22\right\},\left[E_{-} S 23\right\},\left\{E_{-} S 21, E_{-} S 22\right\},\left\{E_{-} S 21, E_{-} S 23\right\},\left\{E_{-} S 22, E_{-} S 23\right\}$ and $\left\{\mathbb{E}_{-} S 21, \mathbb{E}_{-} S 22, \mathbb{E}_{-} \leq 23\right]$. The union of these subsets and the whole elements of $S_{2}$, that is $\left(L_{2_{1}}, L_{2_{2}}\right)$, is a new set. Judge the support functions(P) that the new set can be completed whether satisfy the requirements of $R_{\mathbf{n}}$. The sets that satisfy $R_{\mathbf{z}}$ are:

\{E_P1, E_P2, E_P3, E_S13, E_S21, E_S22, E_S23\},

\{E_P1, E_P2,E_P3, E_S12, E_S13,E_S21,E_S22], [E_P1, E_P2,E_P3, E_S12,E_S13, E_S21, E_S22, E_S23]. Since the third step is the last step, we came to the conclusion through equation (6).

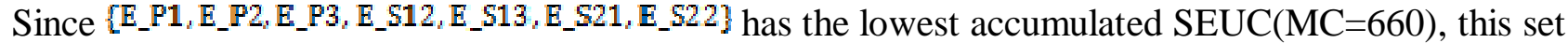
is the optimal support equipment catalogue.

\section{Conclusion}

This paper proposes direct optimization method and dynamic programming optimization method to solve the problem of merging support equipment catalogue. The direct optimization method fast solves the problem of optimizing the support equipment catalogue of simple system with fewer support equipment types; while dynamic programming method solves the optimization problem in the condition of complex system with various support equipment types. The dynamic programming optimization method is verified through an instance. The instance shows that, the dynamic programming optimization method proposed in this paper has an clear optimization target, simple calculation and reasonable optimization result and provides theoretical support for optimizing support equipment catalogue at development phase.

\section{References}

[1] Chen Jun, Chen Yongge, Wang Cheng. Equipment Support Resources Evolution Based on Neural Network Model [ J ]. Academic Journal of MilitaryTraffic Institute, 2008, 10( 4) : 46- 48.

[2] Chen Yonglong, Wang Yuquan, Li Shiying. Study on Determining Operational Support Resources [ J ] . Academic Journal of Institute of Armored Forces Engineering, 2003, 17( 3) : 59- 62. [3] Guo Caifen, Wang Ningsheng. Workstation Equipments Number Optimization Base on Queuing Theory [ J ] . Indutrial Technology economy, 2004, 23( 3) : 101- 102.

[4] Guo Hongfen, Liu Fusheng.Support Equipment Number Optmization Based on Queuing Theory [J]. Academic Journal of Institute of Armored Forces Engineering,.2005,19(1)29-31

[5]Wen Jia, Kang Rui, Liu Rui. Support Equipment Demands Calculation Moded Based on Support Activites[J]. Systems Engineer ing and Electronics, 2010(9):1903-1906.

[6]Wang NaiChao, Kang Rui, Wang Yu. Research on support equipment number determination model basing on item repair policy [J]. Systems Engineer ing and Electronics, 2009(5):1266-1269.

[7]Alfredsson, Patrik. Optimization of multi echelon repairable item inventory systems with simultaneous location of repair facilities [ J] . European Journal of Operational Research, 1997, 99(3) : 584- 595.

[8]Green L, Kolesar P. The point wise stationary approximation for queues with non stationary arrivals [ J ] . Management Science, 1991, 37(1) : 84- 97.

[9]James V.Jones. Integrated Logistics Support Handbook[M]. SOLE LOGISTICS PRESS,2006:13.1-13.2;17.1-17.5

[10]Kang Rui, Kang Xiaoming, Wang Naichao, Study on Support Equipment Types Merging and Optimization 